\title{
Über die Lichtemission von ferroelektrischen Triglyzinsulfatkristallen
}

\author{
G. SCHMidt * und J. Petersson \\ Institut für Experimentalphysik II der Universität des Saarlandes, Saarbrücken, Germany \\ (Z. Naturforsch. 24 a, 1559-1562 [1969]; eingegangen am 4. Juli 1969)
}

\begin{abstract}
Electrical sparks are studied which occur when ferroelectric TGS crystals are heated through the Curie point. The light comes from electrical discharges into the surrounding atmosphere. A rough surface of the crystal and the configuration of the domains have a very strong influence on this effect. Similar sparks are found at the edge of the electrode when the polarization is reversed by an electrical voltage being greater than a certain critical value. A mechanism is proposed which describes these phenomena.
\end{abstract}

In der vorliegenden Arbeit wurden Lichtimpulse untersucht, die beim Erwärmen über den CuriePunkt und beim Umpolarisieren von Triglyzinsulfat (TGS) -Kristallen erzeugt werden. Über die beim Erwärmen auftretenden Lichtimpulse wurde erstmals von ROBERTSON und BAILY ${ }^{1}$ berichtet. Später ${ }^{2}$ wurde von diesen Autoren auf Grund von spektroskopischen Untersuchungen und visueller Beobachtung gezeigt, daß die Blitze auf elektrische Entladungen, die auf der Kristalloberfläche oder in die umgebende Atmosphäre hinein erfolgen, zurückzuführen sind. Eine Wiederholung des Experimentes ist nach diesen Arbeiten innerhalb weniger Minuten nur möglich, wenn die Kristallproben beim Abkühlen einer feuchten Atmosphäre ausgesetzt wurden.

Diese Entladungsvorgänge haben wir bestätigen können. Darüber hinaus haben wir mit den Lichtimpulsen korrelierte pyroelektrische Signale und $\mathrm{Zu}$ sammenhänge mit der Domänenstruktur des Kristalls gefunden. Unsere Experimente lassen sich durch ein einfaches Modell deuten.

\section{Experimentelle Ergebnisse}

1. Mit einem Sekundärelektronenvervielfacher wurden Lichtblitze an unbearbeiteten Kristallstücken, an polierten, unpolierten und künstlich aufgerauhten Kristallplättchen (im folgenden wird darunter ein Plättchen verstanden, das durch einen Schnitt senkrecht zur ferroelektrischen Achse hergestellt wurde) beim Erwärmen über den Curie-Punkt (von ca. $20^{\circ} \mathrm{C}$ auf $60^{\circ} \mathrm{C}$ ) untersucht. Die Lichtabgabe ist besonders stark in der Nähe des Curie-Punktes. Sie

Sonderdruckanforderungen erbeten an: Dr. J. Petersson, Institut für Experimentalphysik II der Universität des Saarlandes, D-6600 Saarbrücken, Universität Bau 4.

* Jetzt: Institut für Metallphysik und Metallkunde der Universität des Saarlandes, Saarbrücken.

1 G. D. Robertson u. N. A. Baily, Bull. Am. Phys. Soc., verschwindet wenige Grade darüber. Die Energie pro Impuls erstreckt sich von ca. $10^{3}$ bis $10^{9} \mathrm{eV}$. Bei schnellem Aufheizen (etwa $1{ }^{\circ} \mathrm{C} / \mathrm{s}$ ) werden weniger, aber energiereichere Impulse beobachtet als bei langsamem Erwärmen (ca. $5 \cdot 10^{-2}{ }^{\circ} \mathrm{C} / \mathrm{s}$ ). Bei den meisten Erwärmungsversuchen lag der Kristall auf einer Metall- oder Glasplatte, deren Temperatur von einem Thermostaten geregelt werden konnte (vgl. Ziff. 3 und 5). Beim Abkühlen treten unabhängig von der Geschwindigkeit innerhalb der Meßgenauigkeit keine Lichtimpulse auf.

2. In der Regel ist die Lichterscheinung um so stärker, je schneller die Kristalle zuvor von hoher Temperatur auf Zimmertemperatur gekühlt wurden und je länger sie auf Raumtemperatur in gewöhnlicher Laboratmosphäre aufbewahrt wurden ${ }^{1}$. Eine Abhängigkeit von dem Feuchtigkeitsgehalt der Luft wurde von ROBERTSON und BAILY ${ }^{2}$ angegeben. Wir stellten fest, daß durch schnelles Abkühlen die Lichtemissionsversuche am gleichen Kristall in kürzeren Zeitabständen (ca. 1 bis 2 Minuten) wiederholt werden können, ohne die sonst nötige Lagerzeit von einigen Stunden einhalten zu müssen.

3. Die sich beim Abkühlen unter den Curie-Punkt ausbildende Domänenstruktur hängt deutlich von der Abkühlgeschwindigkeit ab. Im allgemeinen ist die Domänenstruktur sehr viel grober nach Beendigung der schnellen Abkühlung als nach der langsamen Abkühlung. Abbildung $1{ }^{*}$ zeigt diese durch Ätzmethoden sichtbar gemachte charakteristische Domänenkonfiguration eines Kristallplättchens. Die in Abb. 1 b gezeigte Domänenkonfiguration gleicht den gewöhnlich an TGS sichtbar gemachten Strukturen ${ }^{3}$.

Sol. II, 10, 709 [1965].

2 G. D. Robertson u. N. A. Baily, J. Appl. Phys. 39, 2905 [1968].

* Abb. 1 und 2 auf Tafel 1560 a.

3 F. Jona u. G. Shirane, Ferroelectric Crystals, Pergamon Press, London 1962, S. 47 ff. 
Langsam abgekühlte Kristallplättchen senden beim anschließenden Erwärmen keine Lichtblitze aus. Bei diesen Versuchen lag die Probe auf der Kante eines kleinen Papierwinkels, um jede Wechselwirkung mit der Unterlage zu unterbinden. Diese Ergebnisse lassen einen engen Zusammenhang zwischen der Domänenkonfiguration und der Stärke der Lichtemission vermuten.

4. Die Beschaffenheit der Kristalloberfläche beeinflußt ebenfalls die Lichterscheinung. Kristalle mit einer groben, verschmutzten Oberfläche senden im allgemeinen mehr und energiereichere Blitze aus als solche mit einer glatt polierten, trockenen Oberfläche hoher Reinheit. In dem zuletzt genannten Fall wurde oft überhaupt kein Licht von dem betreffenden Kristall ausgesandt. Wir fanden ferner, daß beim Erwärmen in gesättigter Wasserdampfatmosphäre keine Lichtblitze auftreten. Das ist auch dann der Fall, wenn sich nur die großen Flächen senkrecht zur ferroelektrischen Achse eines Plättchens unter gesättigtem Wasserdampf befinden, also kein Kurzschluß über den Rand des Plättchens besteht. Die Blitze kann man ebenfalls durch eine dicht aufliegende Metallschicht (z. B. Aufdampfschicht) unterdrücken, wobei die Kristallränder durch einen Lack geschützt werden müssen. Aufgebrachte Lackschichten verhindern unabhängig von der Lackart ebenso die Lichterscheinung. Nach Entfernung der Lackschicht läßt sich die Lichterscheinung wieder erzeugen.

5. Bedeckt man die Flächen senkrecht zur ferroelektrischen Achse mit Papierplättchen, so wird die Lichterscheinung an allen untersuchten Kristallen verhindert. Bei sehr stark verletzten Oberflächen mußte das Papier etwas angedrückt werden. Nach einer Erwärmung oder Abkühlung (Temperaturintervall $20^{\circ} \mathrm{C}-60^{\circ} \mathrm{C}$ ) wird das Papier mit Ausnahme von lackierten Papiersorten in der Mehrzahl der Fälle vom Kristall elektrostatisch angezogen.

6. Auf den Oberflächen von Kristallplättchen befinden sich stets mehr oder weniger viele kleine Staubpartikeln oder Kristallite (mikroskopische Beobachtung, Gesichtsfelddurchmesser $1 \mathrm{~mm}$, Teilchengröße ca. $0,02 \mathrm{~mm}-0,2 \mathrm{~mm}$ ). Erwärmt man eine gelagerte Kristallplatte, so beobachtet man, daß sich die Partikeln nach einer gewissen Heizzeit sprunghaft bewegen. Die Bewegung der größeren Partikeln war in der Regel mit einem Knacken verbunden, während kleinere Partikeln oft ganz aus dem Gesichtsfeld heraussprangen. Nach wenigen Sekunden - abhängig von der Heizgeschwindigkeit - sind diese Vorgänge beendet. Dieser Effekt läßt sich im Gegensatz zu den Beobachtungen an Lichtblitzen (vgl. Ziff. 2) nicht durch schnelles Abkühlen wieder reproduzieren. Er tritt vielmehr erst dann auf, wenn der Kristall nach dem Abkühlen entweder mindestens einige Stunden in Laboratmosphäre gelagert oder mit einem feuchten Tuch gerieben wurde. Mit jedem Knacken ist ein energiereicher Lichtblitz $\left(\geqq 10^{7} \mathrm{eV}\right)$ verbunden. Diese Vorgänge (Partikelsprung, Knacken und Blitz) sind also miteinander korreliert und man kann daher das Springen der Partikeln, neben Inhomogenitäten der Oberflächen und der Ränder, als eine der Ursachen für die Entstehung der energiereicheren Lichtblitze ansehen. Insbesondere ergibt sich daraus, daß sich wenige Grade oberhalb des Curie-Punktes keine Partikeln mehr bewegen.

7. Das nun beschriebene Experiment zeigt den Zusammenhang zwischen den Lichtblitzen beim Erwärmen und den dabei auftretenden elektrischen Impulsen. Die Oberflächen eines Kristallplättchens wurden mit Gold bedampft, davon eine Oberfläche nur teilweise, so daß eine freie Randzone entstand. Durch Anlegen eines Gleichfeldes während des Abkühlens wurde das Kristallvolumen unter der kleineren Elektrode einschließlich eines gewissen Randbereiches um diese Elektrode in einer Richtung polarisiert. Bei Zimmertemperatur wurde der Kristall von der Spannungsquelle getrennt und an einen Oszillographen angeschlossen. Beim Aufheizen treten dann energiereiche Lichtblitze zusammen mit pyroelektrischen Impulsen auf. Ohne die Vorbehandlung mit einem Gleichfeld ist die Lichterscheinung deutlich schwächer. Die pyroelektrischen Impulse sind einem Pyrostrom überlagert, dessen zeitlicher Verlauf dem Abbau der spontanen Polarisation des gesamten Kristalls zum Curie-Punkt hin entspricht. Die Impulse und der Pyrostrom haben das gleiche Vorzeichen.

9. Im folgenden werden Lichterscheinungen beschrieben, die beim Umpolen von Kristallen auftreten. Erreicht eine angelegte $50 \mathrm{~Hz}$-Sinusspannung einen kritischen Spitzenwert, der je nach Kristall zwischen $360 \mathrm{~V}$ und $420 \mathrm{~V}$ liegt, so leuchten die Ränder der Elektroden. Die Lichtabgabe beginnt bei etwa $25{ }^{\circ} \mathrm{C}$ und verschwindet dicht am Curie-Punkt. Das in Abb. 2 angeführte Beispiel zeigt, daß bei $35{ }^{\circ} \mathrm{C}$ ein schmaler durchgehender Leuchtsaum den Rand der Elektrode umgibt. Bei steigender Temperatur wird der Leuchtsaum breiter und zunehmend 
G. Schmidt und J. Petersson, Über die Lichtemission von ferroelektrischen Triglyzinsulfatkristallen (S. 1559).

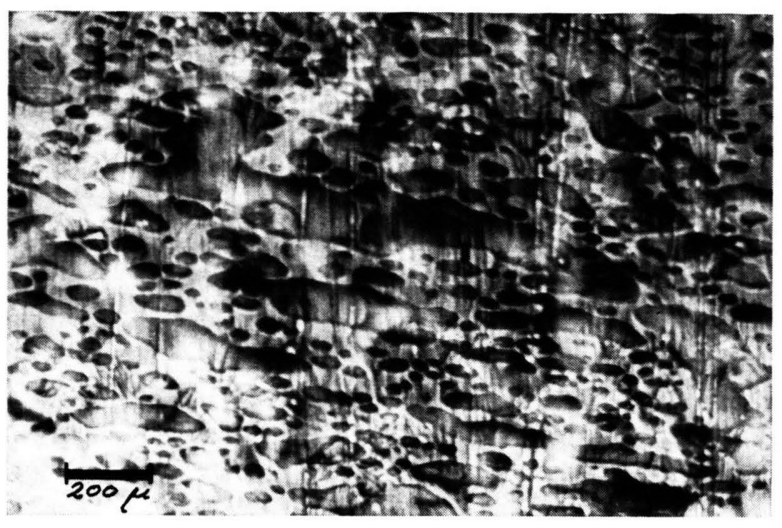

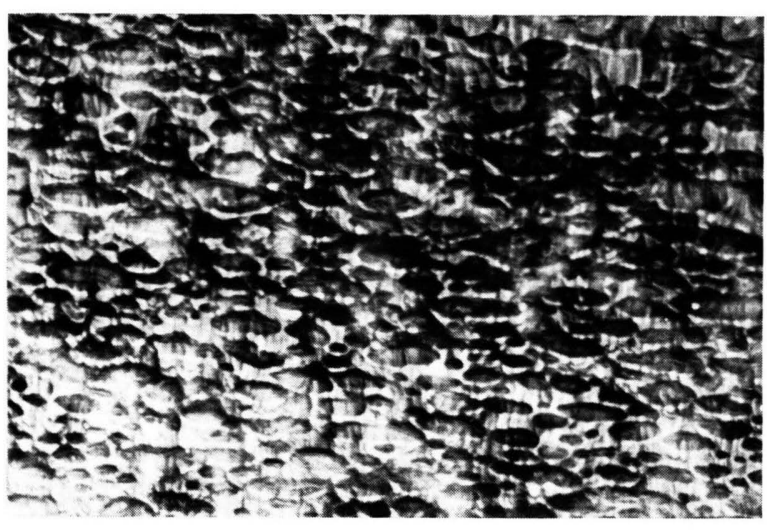

b

Abb. 1. Unterschiedliche Domänenkonfiguration nach verschieden schneller Abkühlung von $60{ }^{\circ} \mathrm{C}$ auf $20^{\circ} \mathrm{C}$. a) schnelle $\left(3^{\circ} \mathrm{C} / \mathrm{s}\right)$, b) langsame $\left(0,05^{\circ} \mathrm{C} / \mathrm{s}\right)$ Abkühlung.

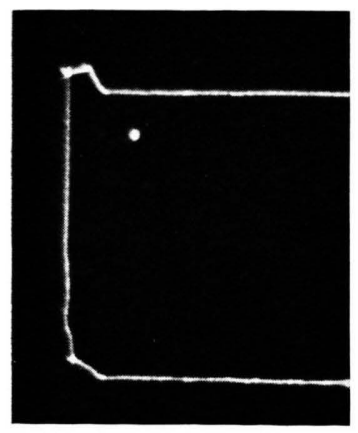

ca. $35{ }^{\circ} \mathrm{C}$

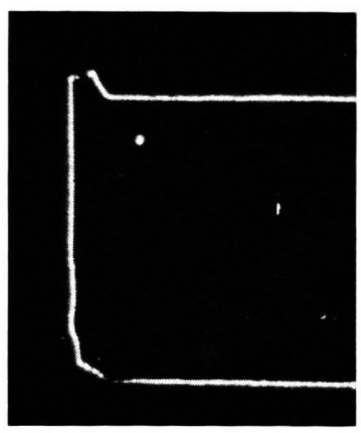

ca. $40{ }^{\circ} \mathrm{C}$

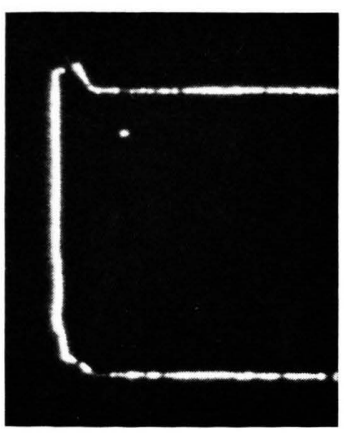

ca. $45{ }^{\circ} \mathrm{C}$

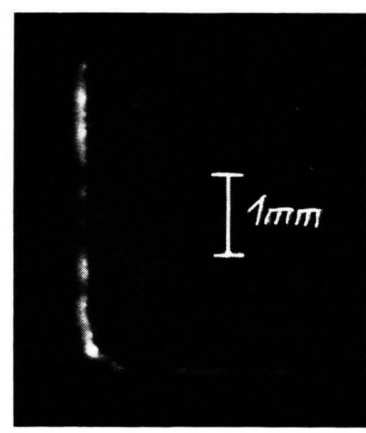

ca. $48{ }^{\circ} \mathrm{C}$

Abb. 2. Lichtemission an den Elektrodenrändern beim Umpolarisieren mit $50 \mathrm{~Hz}$ Sinusspannung. Abstand Kristall-Objektiv ca. $10 \mathrm{~cm}$, Brennweite $5 \mathrm{~cm}$, Öffnung 1:2,0, Film Agfa Isopan Record, Belichtungsdauer ca. 45 Minuten. 

stellenweise unterbrochen. Die maximale Lichtenergie pro Impuls steigt mit der Temperatur an, erreicht etwa $2{ }^{\circ} \mathrm{C}$ unterhalb des Curie-Punktes ein ausgeprägtes Maximum und verschwindet wieder etwa am Curie-Punkt. Dieser Verlauf wird durch eine geänderte Umpolspannung und durch eine geänderte Elektrodengröße qualitativ nicht beeinflußt. Der Wert der kritischen Spannung hängt im Bereich der von uns untersuchten Probendicken zwischen $0,3 \mathrm{~mm}$ und $1,4 \mathrm{~mm}$ fast nicht von der Dicke und der Temperatur ab. Eine an den Kristall angelegte Gleichspannung, deren Anstiegszeit durch einen $R C^{\mathbf{6}}$ Kreis eingestellt werden konnte $(R C$ zwischen $16 \mathrm{~ms}$ und $80 \mathrm{~ms}$ ), führte stets zum Auftreten von Lichtblitzen bei den gleichen kritischen Spannungswerten. Der Schwellenwert ist also unabhängig von der Frequenz.

10. Während der durch Umpolarisieren mit $50 \mathrm{~Hz}$ Sinusspannung hervorgerufenen Lichtemission verdampft die Elektrode an den Rändern und hinterläßt eine verletzte Oberfläche. Stärkere Verletzungen der Oberfläche wurden vor Spitzen und herausragenden Teilen der Elektrode beobachtet. Dies dürfte im Zusammenhang damit stehen, daß an vereinzelten Stellen in der Nähe des Curie-Punktes gröBere Lichtimpulse beobachtet wurden.

11. Die spektrale Zusammensetzung des beim Erwärmen und beim Umpolarisieren erzeugten Lichtes wurde mit Interferenzfiltern im Wellenlängenbereich zwischen $310 \mathrm{~nm}$ und $420 \mathrm{~nm}$ mit derjenigen von kurzen Funken in Luft verglichen. Die gefundenen Transmissionswerte für die verschiedenen Wellenlängenbereiche der Filter zeigten in allen diesen Fällen eine ähnliche Abhängigkeit von der Wellenlänge. Die Intensität des durch Umpolarisieren mit $50 \mathrm{~Hz}$ Sinusspannung erzeugten Lichtes war gerade ausreichend, um mit einem Monochromator mit angeschlossenem Multiplier als Detektor analysiert werden zu können. Um die Lichtemission und somit die Bestrahlungsstärke des Eintrittsspaltes zu erhöhen, wurde die Kristallelektrode zerkratzt, um mehr leuchtende Ränder zu erzeugen. Dennoch mußte der Spalt weit geöffnet werden, wodurch das Auflösungsvermögen auf etwa $12 \mathrm{~nm}$ herabsank. In Übereinstimmung mit den Ergebnissen von ROBERTSON und BAILY ${ }^{1}$, die von ihnen beim Erwärmen von Kristallen gefunden wurden, stellten wir bei unseren Umpolexperimenten stets Emissionsmaxima bei den Linien der zweiten positiven Gruppe des $\mathrm{N}_{2}$, nämlich bei $315,337,357,380$ und $403 \mathrm{~nm}$, fest. Hieraus ist zu schließen, daß die Lichtanregung auch beim Umpolarisieren der Kristalle in der umgebenden Atmosphäre stattfindet.

\section{Diskussion der Ergebnisse}

Während des Abkühlens durch den Curie-Punkt bildet sich die Domänenstruktur aus, was Polarisationsladungen und die damit verbundenen Streufelder auf den Kristalloberflächen zur Folge hat. Diese Polarisationsladungen werden mehr oder weniger schnell durch freie Ladungen kompensiert, wodurch die Streufelder verschwinden. Nach einiger Zeit hat sich auf der Oberfläche senkrecht zur ferroelektrischen Achse eine Flächenladungsdichte $\pm 2 P_{\mathrm{s}}$ aufgebaut. Form und Größe der Domänen werden durch die thermischen und elektrischen Bedingungen während des Abkühlvorganges beeinflußt (vgl. Abb. 1).

Bei einer Erwärmung des Kristalls nimmt die spontane Polarisation ab und die dabei frei werdenden Ladungen werden nun zu Quellen des elektrischen Feldes. Đie Ladungen streben daher eine Neutralisation untereinander an. Bei großer Oberflächenleitfähigkeit oder sehr langsamem Aufheizen überwiegt der Ladungstransport durch Kriechströme ohne elektrische Durchschläge. Bei niedriger Oberflächenleitfähigkeit und passender (grober) Domänenkonfiguration und Oberflächenstruktur kann die Durchschlagsfeldstärke der Luft in den Streufeldern, die sich zwischen den Enden der Domänen auf den Flächen oder an den Rändern der Plättchen ausbilden, überschritten werden. Dies sollte vor allem an kleinen Löchern und Kratzern, am Kristallrand sowie an den Rändern teilweise bedeckender Elektroden der Fall sein. Ebenso können geladene Partikeln entlang der Feldlinien transportiert werden, wobei ein elektrischer Durchschlag in der Luft kurz vor dem Aufprall auf die entgegengesetzte Ladung erfolgt. An Hand dieses Modells kann man verstehen, daß bei einem Kurzschluß in einer Oberfläche senkrecht zur ferroelektrischen Achse eines Plättchens (Wasserdampfatmosphäre, Metallschicht) beim Erwärmen keine Lichtblitze auftreten.

Nach unseren Vorstellungen können also Lichtblitze dann auftreten, wenn eine grobe Domänenstruktur bei Beginn der Erwärmung vorliegt. Abbildung 1 zeigt, daß dies durch eine schnelle Abkühlung unter den Curie-Punkt erreicht werden kann. ROBERTSON und BAILY ${ }^{2}$ gaben als Bedingung für die schnelle Wiederholbarkeit der Erwärmungs- 
experimente an, daß die Abkühlung in einer feuchten Atmosphäre erfolgt sein muß. Trifft unsere Vorstellung zu, so hat man auch in diesem Fall eine grobe Domänenstruktur zu erwarten.

Beim Abkühlen werden keine Blitze ausgesandt, da sich die Domänenstruktur so ausbildet, daß intensive Streufelder vermieden werden. Die Lichtemission wird also begünstigt durch schnelle zeitliche Änderung der spontanen Polarisation (erreicht durch entsprechende Heizgeschwindigkeit), niedere Oberflächenleitfähigkeit sowie eine geeignete Oberflächenbeschaffenheit und eine grobe Domänenstruktur. Das Verhalten beim Umpolarisieren kann wie folgt verstanden werden: Bei erreichter Koerzitivfeldstärke wird nur das Kristallvolumen unter der
Elektrode umpolarisiert. Mit wachsender Spannung werden die nichtmetallisierten Teile durch die hohen Streufelder, vom Elektrodenrand ausgehend, ebenfalls allmählich umpolarisiert. In der Nähe der Elektrode wird daher eine Oberflächenladungsdichte $2 P_{\mathrm{s}}$ frei, die eine elektrische Entladung zum Elektrodenrand hin in Gang setzt. Nach dem Gesagten muß man annehmen, daß diese Entladung in der umgebenden Luft stattfindet, was sich durch die spektroskopische Untersuchung des ausgesandten Lichtes bestätigt hat.

Herrn Prof. Dr. H. E. MüsER möchten wir für die Anregung zu dieser Untersuchung, für die Bereitstellung von Institutsmitteln und für sein förderndes Interesse danken.

\title{
Fluorescence anti-Stokes de $\mathrm{l}^{\prime} \mathrm{Er}^{3^{+}}$et ${\mathrm{du} \mathrm{Tm}^{3+}}^{3+}$ par transfert d'énergie
}

\section{F. Auzel et O. Deutschbein}

\author{
Centre National d'Etudes des Télécommunications, Issy-les-Moulinaux, France \\ (Z. Naturforsch. 24 a, 1562-1568 [1969] ; eingegangen am 10. Juli 1969) \\ Herrn Prof. Dr.-Ing. H. GOBRecht zum 60. Geburtstag gewidmet
}

\begin{abstract}
Antistokes fluorescence in the green and blue region, excited by infrared radiation $(\lambda=0.97 \mu \mathrm{m})$ is observed with a relatively high efficiency in substances which contain not only a luminescent activator $\left(\mathrm{Er}^{3+}\right.$ or $\left.\mathrm{Tm}^{3+}\right)$, but also a sensitizer which has a strong infrared absorption $\left(\mathrm{Yb}^{3+}\right)$. Polycrystalline mixed tungstates and germanate glasses have been studied. The green fluorescence of $\mathrm{Er}^{3+}$ increases as a square-law function of the excitation intensity, the blue fluorescence of $\mathrm{Tm}^{3+}$ as a cubic function. This effect has been used to build an optical amplifier which has served to measure the excitation spectra. The mechanism of excitation is the following: Absorption of the infrared radiation by the sensitizer, followed by energy transfers to the luminescent activator, which is excited stepwise up to the initial level of luminescence. Oscillator strengths for some transitions between excited levels have been calculated.
\end{abstract}

\section{INTRODUCTION}

D'après la loi de Stokes, l'énergie des quanta d'excitation de fluorescence doit être supérieure à celle des quanta d'émission. Néanmoins, en présence d'une densité de phonons suffisante, on observe des raies anti-Stokes, mais la différence d'énergie est de l'ordre de $k T$, donc à la température ambiante de quelques centaines de $\mathrm{cm}^{-1}$ environ.

Une autre sorte de fluorescence anti-stokes (A.S.), avec des différences d'énergie plus grandes est à la base des compteurs quantiques proposés par BlOEMBERGEN ${ }^{1}$. Des photons de «pompage» de fréquence $v_{1,2}$ provoquent dans un matériau approprié des

Reprint requests to Prof. Dr. O. Deutschbein, Centre National d'Etudes des Télécommunications, 3 avenue de la Republique, Issy-les-Moulineaux (Seine), France. transitions $E_{1} \rightarrow E_{2}$, et ensuite les photons de «signal» de fréquence $v_{2,3}$ la transition $E_{2} \rightarrow E_{3}$. On observe ensuite une fluorescence de fréquence $v_{\mathrm{fl}}=v_{1,2}+v_{2,3}$ correspondant à la transition radiative $E_{3} \rightarrow E_{1}$. Cependant, le rendement de ces compteurs quantiques est généralement très faible.

L'un de nous a montré pour la première fois ${ }^{2}$ et ${ }^{3}$ qu'on peut obtenir des rendements beaucoup plus grands si l'on utilise des matériaux qui contiennent deux terres rares, dont l'une sert de sensibilisateur et l'autre de système fluorescent. L'excitation a lieu par absorption successive de plusieurs photons par le sensibilisateur et par transfert d'énergie sur le système fluorescent.

1 N. Bloembergen, Phys. Rev. Letters 2, 84 [1959].

2 F. Auzel, C. R. Acad. Sci. Paris 262 B, 1016 [1966].

${ }^{3}$ F. Auzel, C. R. Acad. Sci. Paris 263 B, 819 [1966]. 